\title{
Using Emergence Phenomenon in Meaningful Image Segmentation for Content-based Image Retrieval
}

\author{
Sagarmay Deb \\ Central Queensland University, 400 Kent Street, Sydney 2000, NSW \\ Australia
}

\section{Introduction}

Research on multimedia systems and content-based image retrieval has gained momentum during the last decade. Content-based image retrieval (CBIR) is a very difficult area in the access of multimedia databases simply because there still exist vast differences in the perception capacity between a human and a computer. There are two basic problems that still remain unresolved in the area although some progresses have been made [13]. The first one is the problem of efficient and meaningful image segmentation where we break-up a particular image into meaningful parts based on low-level features like color, texture, shape and spatial locations. Developing a segmentation algorithm which will meaningfully segment all images is yet an open problem in image analysis [8]. The second one is the vast gap existing for an image between low-level features mentioned earlier and high-level or semantic expressions contained in the image like the image of a car, a house, a table and so on [11]. To develop efficient indexing techniques for the retrieval of enormous volumes of images being generated these days, we need to achieve reasonable solutions to these abovementioned two problems. But only in very limited and selected cases, some kinds of solutions have been achieved with apparently promising experimental results. In this paper we focus our attention on the first problem. The research identifies few issues causing this gap, for example, failure to capture local image details with low level features, unavailability of semantic representation of images, inadequate human involvement in the retrieval, and ambiguity in query formulation [9]. We offer future directions of research in solving this difficult problem using emergence phenomena.

Section one gives an introduction of the area. Section two provides a definition of emergence phenomenon. Section three talks about the use of emergence phenomenon in extracting meanings in image segmentation. Section four suggests future directions of research. We put our concluding remarks in section five.

\section{Emergence phenomenon}

\subsection{Definition of emergence phenomenon}

A feature of an image which is not explicit would be emergent feature if it can be made explicit. There are three types of emergence: computational emergence, thermodynamic 
emergence and emergence relative to a model [1]. In computational emergence, it is assumed computational interactions can generate different features or behaviors [3],[7]. This is one of the approaches in the field of artificial life. Thermodynamic emergence is of the view that new stable features or behaviors can arise from equilibrium through the use of thermodynamic theory. In emergence relative to a model, deviation of the behavior from the original model gives rise to emergence. We will use this latter view in our work.

In computational emergence, new shapes or images develop but within certain limit as programmed by the computer programmers. No new shape can emerge beyond the logic of the program.

In thermodynamic emergence, emergence can be defined as emergence of order from noise. Stochastic processes at micro-level form discrete macro-level structures or behaviors. The example of this type of emergence is gas where stochastic movements of atoms or molecules within the gas create the ordered properties of temperature, pressure and volume at a higher level.

Example of emergence relative to a model is where changes in internal structure and consequently in its behavior occur and we as observers will need to change our model to track the device's behavior in order to successfully continue to predict actions. The example of a square having two triangles hidden in it as given below is of this type.

Whenever we shift our focus on an existing shape in other words an image, new shape emerges. The representation of the new shape is based upon view of the original shape. The new shape emerges as we change our view of the original shape. This is the fundamentally most important idea of emergence.

Two classes of shape emergence have been identified: embedded shape emergence and illusory shape emergence. In embedded shape emergence all the emergent shapes can be identified by set theory kind of procedures on the original shape under consideration. For example, in a set $S=\{a, b, c, d, e\}$, we can find subsets like $S 1=\{a, b, c\}, S 2=\{c, d, e\}, S 3=\{a, c$, e) and so on. But in illusory shape emergence, where contours defining a shape are perceived even though no contours are physically present, this kind of set theory procedures are not enough and more effective procedures have to be applied to find these hidden shapes [4],[5]. These procedures could be based on geometrical, topological or dimensional studies of the original shape.

\subsection{Structure, behavior and function of emergence}

Structure of a shape is the physical definition of the shape. For example, a box could be rectangular in shape, its length, width and height as well as color, substance like wood, metal or hard paper would define the structure of the shape. Behavior of the box could be to contain certain stuffs in it and the function could be to carry stuff from one place to another using the box as a container, which is the purpose for which the box is used. Emergence of new structure, behavior or function takes place when these descriptions are interpreted in ways not anticipated in the original description [4].

\subsection{Examples of emergence}

Shape emergence is associated with emergence of individual or multiple shapes. The following figures are examples of shape emergence. 


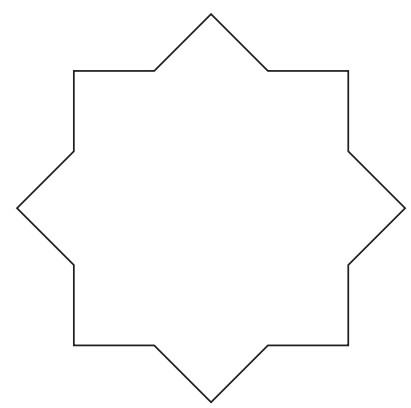

The Existing Shape
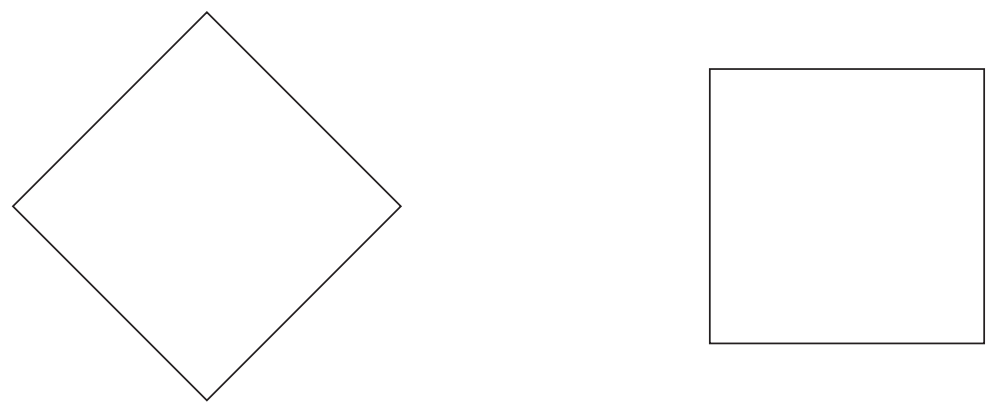

Fig. 1.1. Two emergent shapes derived from the existing one [5]

\subsection{Definition of emergence index}

Image retrieval where the hidden or emergence meanings of the images are studied and based on those hidden meanings as well as explicit meanings, an index of search is defined to retrieve images is called emergence index.

When images are retrieved based on textual information, various parameters and descriptions might define the input and the images of the database. Whenever there would be symmetry of parameters and descriptions, the image could be retrieved. In CBIR, color, texture and shape are widely used as index to retrieve images. But in our studies, we can find the hidden meanings of the images and whenever those hidden meanings match with the input given, although the original image may not match at all with the input, we can retrieve that image.

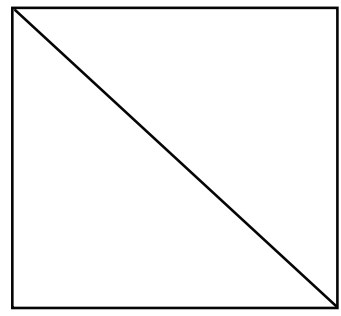

Fig. 1.2. Example of emergence where diagonal generates two triangles 
To describe in detail, as we have mentioned, emergence is a phenomenon where we bring out the shapes, which are not explicit but implicit. The above figure shows a simple example of emergence where an apparently square shape has two triangles hidden in it as we discussed earlier.

When an input would come in the form of an image, the image could be studied based on features, constraints, variables, domains and emergence and converted into parametric form. Then the image database would be accessed and each image would be interpreted considering the items mentioned above and converted into parametric form like the input image. Whenever there would be a match between parameters of the input and the images of the database, those records would be selected. In other words, indexing would be decided by the outcome of emergence which means more meaningful images could be found hidden in an image which would otherwise not be understood.

As we have mentioned earlier, many images of the database may not have any apparent similarities with the input, but emergence could bring out the hidden meaning of the image and could establish similarities with the input image. So emergence outcomes of the images would form the index structure of the search.

\subsection{Structure of emergence index}

\subsubsection{Parameter definitions}

To make an effective query, the images in the query or database must be analysed so that we know what we are looking for and where to look for. Features, domains, variables, constraints, similarities, indexing are the parameters, which play very important role in similarity searching. Hence they constitute the structure of the emergence index.

\section{Features}

Input, as we mentioned earlier, could come in the form of text. For example, the text may indicate we have to pick up all the images of a database that contains the image of a particular person or an object. In this case, nothing much could be done on input side in the sense that we cannot study the input's features etc. We have to go through the images and pick up the image records. But if the query comes in the form of an image of a person then we have to analyse it before accessing the database images. Features would tell us about a few important characteristics of the input image. To start up, our query could be an image where a particular person is sitting on a chair. Then obviously there are two important features in the query image - the particular person and the chair. We have to locate these two features in the database image while searching for similarities.

We know color plays a very important role in the definition of features. Quite often a query might mention an object with certain specified color to be picked up from the databases. Besides, color is being used extensively in various models as a tool in finding symmetry with the input image.

Sometimes the input may be in the form of a sketch. In that case, a similar kind of image should be selected from the image database. The selection should be made on the basis of few dominant characteristics of the input and image and finding similarities in those characteristics.

Texture is another part of the feature where the general alignment of the image like the background part of the image is considered where the image of a person or an object could be the dominant part. 
There would be some global features of an image like area, perimeters and a set of rectangles or triangles that cover the entire shape and there would be some local features, which can be obtained from the image's local region.

When we retrieve images by browsing, then in most cases the user does not have clear idea about what he or she is looking for. In this case, there would not be input image and the search through browsing would be manual. The user would have vague idea about the features of the images to be retrieved like the picture of a particular person with certain specified background. In the objective features based queries, the retrieval is performed on an exact match of attribute values whereas in the subjective features, query is specified by features, which could be interpreted differently by different users.

Retrieval by motion facilitates retrieving spatio-temporal image sequence depicting a domain phenomenon that varies in time or geographic space.

Again, sometimes images are retrieved, as we mentioned earlier, by text. In other words, text defines the features of the images to be selected. Then the database is searched based on those features defined in the input [6].

\section{Domain}

We now proceed to discuss the domain. Domain is a sort of classification of the images into certain categories. Domain is a way for a class of objects to present knowledge representing a certain concept held by objects [10].

We can make use of the various properties of the features of an image to define the domain in which the image concerned would lie. For example, from an image we can understand whether the image is that of a geographical location of a certain area of the earth or the image is that of a person or object with certain background like a screen or a landscape behind. This kind of classification at the initial stage of search should enable us to access the image database rapidly and more efficiently.

Also in a multimedia database, the database might contain various kinds of records like the images, the data in figures, the documents, audios, videos and so on. The concept of domain would classify them according to their categories.

The domain could also be formulated based on the length of the certain features of the image like finding the images of a particular person or object where the length is of certain range value. Color could also define a domain where the images having a particular color or a combination of colors lie in one domain. Domain could be defined on the basis of objects only. For example, we can pick up image where the images of a triangle and square would be present. We can make it more specific by mentioning color and length or size of the triangle and the rectangle to pick up images like where the triangle is red colored and sides of length, say, $(3,3,3)$ and rectangle of color white and sides of length, say, $(4,2)$.

\section{Variables}

Since in our research we are considering a multimedia database where a particular record in the database could be an image or a data record or a document and so on, the definition of variables would vary depending upon the type of records we are considering.

In an image database, where we would consider two-dimensional pictures, the image of an object or a person have to be measured. If we try to measure it graphically, then the size could be measured in terms of $x$ and $y$ coordinates. Therefore, size would be a very important variable in our research. 
Color could be another very important variable. There are, as we know, many colors available and for specific definition of the image, the color would play a very vital role. It is possible to define colors digitally in the sense that each color could be given a digital number like red $=1$, blue $=2$ and so on.

Location would point towards where a particular object of interest is situated. If we were interested in finding the image of a person with a certain background at a particular point of the image, then location would tell us about where it is present. In a graphically defined image, the coordinate of the center of the image would give information about the location of the object.

Distance between two particular objects of interest is a variable to be considered. Sometime in an image depicting a geographic picture, the distance between two points is very important. Here also we should be able to measure the distance between two objects by applying graphical methods.

Motion of objects, like storm or cloud moving in a satellite picture, is also an important variable. We have to measure the distance travelled by the object in the picture and then convert it into kilometers and notice the time difference. From these we can measure the speed, velocity and so on of the object.

\section{Constraints}

It could be a very good idea to define an image in terms of various constraints in the sense that constraints help define the image more specifically.

In our case of multimedia database, where various kinds of data could be there, the concept of constraints is very important. For example, if the image is that of a rectangle, we know one of the constraints would be that the number of sides of the object is 4 . Then the second constraint would be opposite sides are parallel. The third one would be opposite sides are equal. If we include the emergence phenomenon, then if there is a diagonal drawn on it, this would give rise to two triangles. These constraints together could define the image successfully.

In an image of a geographical map of any part of the world, the concept of constraint would be effective in finding the location. If we are interested in finding a place, for example, an island with triangular shape, then obviously the constraint would be number of sides is 3 . If we have more information about sides like whether any two sides are same or all sides are same or all sides are of different size, then this kind of information should help us identify the object more accurately.

\section{Similarities}

In a database containing only data, the input may be a query with certain constraints like to pick up records from a SALARY database where salary is greater than, say, 30000. In relational database, as we know, this can be accomplished by a SQL command with the following kind of statement:

\section{SEL * FROM SALARY_DB WHERE SALARY > 30000.}

This would pick up all the records with salary $>30000$.

In our multimedia database system, this kind of queries could also be made and we can handle them with this kind of or more complicated SQL statements.

But when the input is in the form of image, then we have to find the similarities of the input in the image part of the database. The basic approach to the problem of similarity is to find 
certain parametric values as well as some coordinates of the input image. Then we find the same for various image of the image database and pick up records where some matching occurs. Of course, we study the emergence phenomenon in both input and images of an image database while calculating parameters. For example, if we want to find similarities involving a triangular figure as input, then some of the parametric values could be defined like, number of sides which is 3, length of each sides, color of the triangle. Based on these values and constraints, we can find out similarities in the image database. But in the image database, there could be figures like squares or rectangles with a diagonal drawn on them. Then obviously this diagonal gives rise to two triangles according to emergence. So we have to study these cases too, find out the parameters of these triangles to see whether they match our parameters from the input.

\section{Indexing}

In the early stage of data processing, there was no established conception of indexing. Most of the data files were accessed sequentially. This was pretty slow and inefficient particularly when the data file is big enough. To get rid of this problem, the concept of indexing came to the picture. At the initial stage, a number is used to be given against each record by the system in a file created on disk. We could specify these numbers to access any record randomly. Then came the concept of Indexed Sequential Access Method where instead of assigning separate number against each record, a field or a combination of fields were started being used as key. There could be two kinds of indexing, one where the key value in a particular file is unique and the other where the key value could be duplicate. The search method is called Binary Search where to find a particular key value, the whole file is divided into two halves and the part, which contains the particular key value we are searching, is taken and then divided into two halves again. The part here which contains the key value is again taken and divided into two halves. This process continues until it finds the match against the key value.

Nowadays an old file system is hardly used in maintaining computer records. Instead, a database system has been developed. The latest development in this field is Relational Database System, which contains tables to store data.

In our problem of dealing with multimedia databases, which would contain images, the concept of indexing is very important. We are trying to develop a more sophisticated method of indexing where there won't be any clear-cut definition of index against the images, but indexes would be defined based on our study of emergence phenomenon of each of the image. Sometime to locate a particular spot in the geographic map of a part of the world, an input image would point to a particular part and that particular part in one or more than one image could be the outcome of emergence or it could be straight away present in the map without any emergence. In either case, input image refers to an index, which is nothing but that particular spot of the map.

\subsubsection{Model of the emergence index}

Emergence indexes can be defined out of five factors as discussed in section 2.5.1.

$$
E I=f(D, F, V, C, E)
$$

Where $E I$ stands for emergence index, $D$ for domain where the image belongs, $F$ for features, $V$ for variables which can define the feature's constraints under which the features are defined, $C$ for constraints and $E$ for emergence characteristics of images. 
We believe any image, static or in motion, could be expressed semantically in terms of the above mentioned five parameters.

\section{Construction}

We take the case of a square with a diagonal, as mentioned earlier, to build up an emergence index. If this is an image in the database, then firstly we have to put it under certain domain $D$ for the ease of accessing. Since images are generated in enormous volume, we have to put them in various separate entities or tables according to certain classification rather than in one table which could be extremely time consuming to access. The table that would contain this square image record would define the domain of the image. We can term it as TAB1.

To define the second factor $F$, we find the number of maximum sides present would be 5 , where there are 4 regular sides and 1 diagonal.

The variables are $a, b, c, d, e$ where first four define the perimeter of the square and $e$ the diagonal.

The constraints $c$ are $a=b=c=d$ since it is a square.

The emergence $E$ is composed of two triangles with sides $a, b, e$ and c, d, e.

Hence Emergence Index

$$
E I=\{T A B 1 ; 5 ; a, b, c, d, e ; a=b=c=d ;(a, b, e \text { and } c, d, e)\}
$$

\section{Use of emergence phenomenon}

We provide a very simple example of how emergence phenomenon can give rise to meanings for a segmented image where apparently no meaning exists.

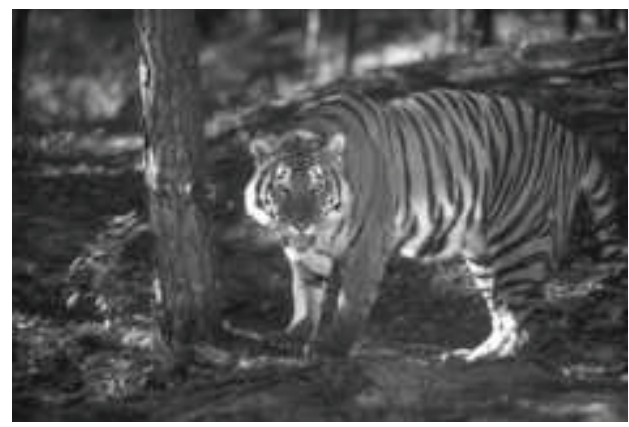

Fig. 1.3. The image of a tiger in a picture before segmentation [12]

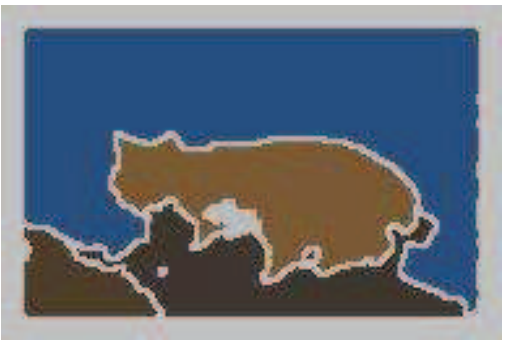

Fig. 1.4. The image of the tiger after segmentation [12] 
Here we see in Figure 1.3 the image is very clearly of a tiger. We segment the while picture and get the image of the tiger in Figure 1.4. But we note that the figure does not clearly point to the image of the tiger but indicates an animal. So we can interpret the image of the tiger in Figure 1.4 as that of an animal instead of a tiger. This is the outcome of emergence phenomenon where we are able to interpret the segmented image in a different way than original picture shows or in other words we extract different meaning of the segmented image. This would enable us to ascribe some meanings to segmented image which otherwise after segmentation may not deliver any meaning. This way through emergence phenomenon we interpret segmented image in a meaningful way.

\section{Suggested future directions of research}

There are various attempts made to extract semantic meanings from an image to fill-in the semantic gap between low-level features and high-level semantic meanings which can arise from image segmentation. These include Latent Semantic Indexing (LSI), contextual search, user feedback, data clustering in the extraction of perceptual concepts, content-based soft annotation (CBSA), image classifications, ontology, top-down, ontologically driven approaches and bottom-up, automatic-annotation approaches, using machine learning methods to associate low-level features with query concepts, using relevance feedback to learn users' intention, generating semantic template to support high-level image retrieval, fusing the evidences from HTML text and the visual content of images for WWW image retrieval, use of ontology which represent task-specific attributes, objects, and relations, and relate these to the processing modules available for their detection and recognition, use of context-awareness for identifying image semantics and relationships to contribute to closing the semantic gap between user information requests and the shortcomings of current content-based image retrieval techniques, enhanced ICBIR system which allows users to input partial relevance which includes not only relevance extent but also relevance reason for a multi-phase retrieval where partial relevance can adapt to the user's searching intention in a from-coarse-to-fine manner [2].

Although these are good, constructive progresses in solving the problem of semantic gap in CBIR, they cannot define the semantic meanings of an image specifically. They can contribute to some broad classification of the image in certain groups.

To solve this problem we have to develop devices to define the semantic meanings of an image very specifically from low-level features and that should be done automatically without users' interaction. We seem to be still far away from this objectivity.

As we have shown in Section 3 emergence phenomenon can bring meanings out of a segmented image where apparently no meaning could be found. We plan to work on the theory of emergence index as described in Section 2 in future to generate softwares to provide the assistance in identifying the images from a segmentation.

\section{Conclusion}

In this paper we studied the problem of extracting meaningful image segmentation using emergence phenomenon. We plan to continue our work in this area to extract meanings from a more complex image segmentation where it is really difficult to find any meaning from the segmented image. Since this is a very important area of research with major 
implications in all spheres of life beginning with medical images, the necessity of this study cannot be overestimated.

\section{References}

[1] Cariani, P. (1992). Emergence and Artificial life, in Langton, C., Taylor, C., Farmer, J.D. and Rasmussen, S. (eds), Artificial Life II, Addision-Wesley, Reading, pp. 775-797

[2] Deb, S. (2010). Using Relevance Feedback in Bridging Semantic Gaps in Content-based Image Retrieval, The Second International Conference on Advances in Future Internet (AFIN2010), Venice/Mestre, Italy, July 18 - 25, 2010

[3] Forrest, S. (ed.) (1991). Emergent Computation, Elsevier, New York

[4] Gero, J. S. and Maher, M. L. (1994). Computational Support for Emergence in Design, Information Technology in Design Conference, Moscow, September 1994

[5] Gero, J. S. (Year Unknown). Visual emergence in design collaboration, Key Center of Design Computing, University of Sydney

[6] Gudivada, V. N. and Raghavan, V. V. (1995). Content-Based Image Retrieval Systems. IEEE, September 1995

[7] Langton, G .L. (1989). Artificial Life, Addision-Wesley, Reading

[8] Mehta, D., Diwakar, E.S.V.N.L.S., Jawahar, C.V. (2003). A Rule-based Approach to Image Retrieval, www.iiit.net/techreports/2003_8.pdf

[9] Reducing semantic gap in content based image retrieval using region based relevance feedback techniques, (2006), http:/ / www.gscit.monash.edu.au/gscitweb/seminar.php?id=41

[10] Yoshitaka, A., Kishida, S., Hirakawa, M. (1994). Knowledge-Assisted Content-Based Retrieval for Multimedia Databases, IEEE Multimedi,Winter 1994, pp.12-21

[11] Zhau, R., Grosky, W.I. (2002). Bridging the semantic gap in image retrieval, http:/ / citeseer.ist.psu.edu/497446.html

[12] Image Segmentation, www.cs.toronto.edu/ jepson/csc2503/segmentation.pdf

[13] Bridging the Semantic Gap in Image Retrieval http:/ / citeseer.ist.psu.edu/497446.html 


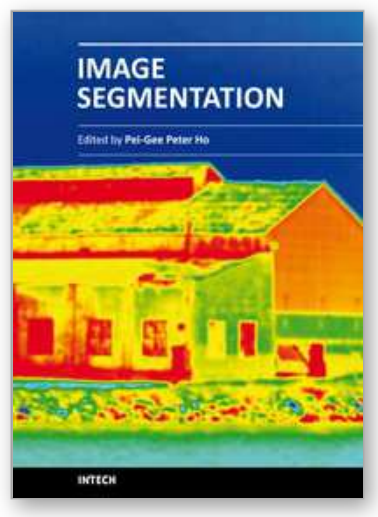

\author{
Image Segmentation \\ Edited by Dr. Pei-Gee Ho
}

ISBN 978-953-307-228-9

Hard cover, 538 pages

Publisher InTech

Published online 19, April, 2011

Published in print edition April, 2011

It was estimated that $80 \%$ of the information received by human is visual. Image processing is evolving fast and continually. During the past 10 years, there has been a significant research increase in image segmentation. To study a specific object in an image, its boundary can be highlighted by an image segmentation procedure. The objective of the image segmentation is to simplify the representation of pictures into meaningful information by partitioning into image regions. Image segmentation is a technique to locate certain objects or boundaries within an image. There are many algorithms and techniques have been developed to solve image segmentation problems, the research topics in this book such as level set, active contour, AR time series image modeling, Support Vector Machines, Pixon based image segmentations, region similarity metric based technique, statistical ANN and JSEG algorithm were written in details. This book brings together many different aspects of the current research on several fields associated to digital image segmentation. Four parts allowed gathering the 27 chapters around the following topics: Survey of Image Segmentation Algorithms, Image Segmentation methods, Image Segmentation Applications and Hardware Implementation. The readers will find the contents in this book enjoyable and get many helpful ideas and overviews on their own study.

\title{
How to reference
}

In order to correctly reference this scholarly work, feel free to copy and paste the following:

Sagarmay Deb (2011). Using Emergence Phenomenon in Meaningful Image Segmentation for Content-based Image Retrieval, Image Segmentation, Dr. Pei-Gee Ho (Ed.), ISBN: 978-953-307-228-9, InTech, Available from: http://www.intechopen.com/books/image-segmentation/using-emergence-phenomenon-in-meaningfulimage-segmentation-for-content-based-image-retrieval

\section{INTECH}

open science | open minds

\author{
InTech Europe \\ University Campus STeP Ri \\ Slavka Krautzeka 83/A \\ 51000 Rijeka, Croatia \\ Phone: +385 (51) 770447 \\ Fax: +385 (51) 686166 \\ www.intechopen.com
}

\author{
InTech China \\ Unit 405, Office Block, Hotel Equatorial Shanghai \\ No.65, Yan An Road (West), Shanghai, 200040, China \\ 中国上海市延安西路65号上海国际贵都大饭店办公楼405单元 \\ Phone: +86-21-62489820 \\ Fax: +86-21-62489821
}


(C) 2011 The Author(s). Licensee IntechOpen. This chapter is distributed under the terms of the Creative Commons Attribution-NonCommercialShareAlike-3.0 License, which permits use, distribution and reproduction for non-commercial purposes, provided the original is properly cited and derivative works building on this content are distributed under the same license. 